\title{
Field Emission Organic Light Emitting Diode
}

\author{
Meiso Yokoyama
}

Additional information is available at the end of the chapter

http://dx.doi.org/10.5772/52487

\section{Introduction}

Several flat panel displays (FPDs) technologies, such as liquid crystal displays (LCDs), plasma display panels (PDPs), light-emitting diodes (LEDs), organic light-emitting devices (OLEDs) and field emission displays (FEDs), have been developed. They coexist because each technology has its own unique properties and applications.

In recent years, the developments in the OLEDs have gradually reached very advantageous of existence. These advantageous characteristics include self-luminous, wide viewing angle and low power consumption, etc. which make OLEDs very useful for numerous display applications and lighting devices. To effectively improve the characteristics of an OLEDs, there are many ways to be adopted. Such as: (a) structure: using the quantum well structure or multilayer structure to enhance efficiency by promoting the radiative recombination capability; (b) material: use of a low work function metal as the cathode or a high carrier mobility material to allow efficient carrier injection into OLEDs structure; (c) doping: by doping the guest material into the host material to increase the efficiency of recombination, such as phosphorescent sensitizer.

However, the performance of OLEDs using above methods will be limited. Therefore, in this chapter, we propose new FEOLEDs with electron multiplier.[1,2-5, 6]

An effective enhancement in the lighting efficiency is achieved by using the external electron source supplement into the OLEDs. The FEOLEDs can simply be divided into two types: FEOLED (original diode type) and triode type. The structure of FEOLEDs is similar to that of the field emission diodes (FEDs), but formers utilize an organic EL light emitting layer instead of an inorganic phosphor thin film used in FEDs. The mechanism of operation of FEOLEDs is the same as OLEDs.

In FEOLEDs also a hole blocking layer is used to confine the electron-hole pairs to enhance recombination in the organic light emitting layer. Besides, to avoid damage to the organic 
material by electron beam bombardment, an aluminum (Al) thin film is coated on the organic light emitting layer facing to the carbon nanotubes (CNTs) template to protect the organic light emitting materials and thereby enhance the luminous efficiency of the FEOLEDs. In a triode FEOLED an electron multiplier layer is inserted between the anode and cathode. This layer amplifies the field emission electrons and then injects them into the organic light emitting layer.

In this chapter, as stated above, we have proposed and discussed two kinds of electron multipliers: 1) a dynode and 2) a strip electron multiplier. The dynode is formed between the cathode and organic light emitting layer to provide electron amplification capability as well as it makes a FEOLED more stable. The electrons emitted from the cathode move towards the dynode as they are attracted by the applied electric field. The primary electrons impact the secondary electron material of dynode to produce the secondary electrons. Finally, both primary and secondary electrons are directly injected into the organic light emitting layer and increase the current density of FEOLEDs. Due to the presence of a dynode it is difficult to fabricate very thin FEOLEDs. Therefore, the strip electron multiplier has been proposed as it can easily be incorporated in FEOLEDs. The strip electron multiplier is formed on the organic light emitting layer facing to the CNTs cathode, and made by strip Al coated with secondary electron material (Cesium iodide, CsI). CsI does not completely cover the strip Al.

The mechanism of electron amplifier ability of the strip electron multiplier is the same that of a dynode, but the process of fabrication is easier. The organic light emitting layer of FEOLEDs integrated with the strip electron multiplier forms an OLEDs, which can operate independently. Accordingly, applying an electric field to the CNTs template and strip electron multiplier one can attract electrons to impact the strip electron multiplier to generate the secondary electrons. Therefore, the current density of OLEDs is increased by supplementing the electrons into the multilayer of the organic light emitting layer. In this way, the luminance efficiency of FEOLEDs with strip electron multiplier can further be enhanced by one and a half times more than of OLEDs.

The organization of this chapter is as follows. First the concept and mechanism of operation of FEDs and OLEDs are introduced. Then are illustrated the basic concepts and luminescent mechanisms of FEOLEDs and experimental procedure of fabricating them, FEOLEDs of diode structure and triode structure and their characteristics of electron multiplier are discussed next. Finally, we discuss the advantages and disadvantages of the conventional OLEDs and novel FEOLEDs, including some suggestions for future work..

\section{Field emission light emitting diodes (FEDs)}

A FED is a vacuum electron device, sharing many common features with the vacuum fluorescent displays (VFDs) and cathode ray tubes (CRTs) [7]. Like in a VFDs or CRTs, the image in a FED is created by impacting electrons from a cathode onto a phosphor coated screen. In a CRTs the electron source is made up of up to three thermionic cathodes [8]. A 
set of electromagnetic deflection coils raster the electron beam across a phosphor screen, which is typically held at a potential of $15-30 \mathrm{kV}$ [9]. In a FED the electron source consists of a matrix-addressed array of millions of cold emitters. This is field emission arrays (FEAs), which is placed in closed proximity $(0.2 \mathrm{~mm})$ to a phosphor faceplate and is aligned such that each phosphor pixel has a dedicated set of field emitters [10].

The idea of a FED dates back to the 1960s, when Ken Shoulders of the Stanford Research Institute (SRI) proposed electron beam micro devices based on FEAs [11]. The first operating FEAs were demonstrated by Capp Spindt, also of SRI, in 1968 [12]. Despite many advantages of the spindt-type FEA fabrication technique, scaling this method to large area substrates ( $>400 \mathrm{~mm}$ on the side) is still a major challenge. Another difficulty associated with the scale up of spindt process is the large size of the evaporator required to deposit the spindt tips. Most phosphors have low luminous efficiency at voltages below $3 \mathrm{kV}$ because of the low electron penetration depth and high, non-radiative recombination rates at the surface. While raising the emission current density increase the brightness, high current density leads to faster aging of the phosphor, thus further decreasing the brightness [13].

Among various kinds of emitters in field emission devices, carbon nanotubes (CNTs) have been attracting a considerable attention due to their excellent field emission characteristics of high field emission current density and low turn-on electric field [14]. In order to enhance the field emission electron ability and emission uniformity in large area CNT-FED panels, additional methods are required to improve uniformity by inserting the gate design for electron multiplier and focusing. A gate coated with the secondary electron emission (SEE) materials for obtaining electron amplification is called a dynode [15]. In general, any insulator with low work function is suitable for SEE application [16]. The mechanism of dynode can be simplified by the following processes: (i) the primary electrons penetrate into a certain depth of an insulating layer; (ii) through collision, the energy of the primary electrons is transferred to the bound electrons of the insulator, leading to a release of electrons; (iii) the released electrons migrate to the surface and escape into the vacuum as secondary electrons.

Therefore, the field emission involves the extraction of electrons from a solid by tunneling through the surface potential barrier. The emitted current depends directly on the local electric field E at the emitting surface and metal's work function $(\Phi)$ as shown in Fig.1 [17]. The field-emission properties of wide band gap materials (WBGMs) is favorable for the emission, as it is considered a property unique to the surface of emitter [18]. The role of the WBGMs in CNTs field emission is to decrease the effective work function of emitters, which increases emissivity. We can assure that the carbon nanotubes are excellent electron sources, providing a stable current at very low fields and capable of operating in moderate vacuum.

In summary, the light emitting principle of FEDs is that the electrons are excited and accelerated by the high electric field under vacuum, so as to become sufficiently energized to bombard the inorganic phosphor to emit light. Although CNT-FED was very successful in achieving the result in different low voltage phosphors such as $\mathrm{ZnGa}_{2} \mathrm{O}_{4}+\mathrm{In}_{2} \mathrm{O}_{3}, \mathrm{ZnO}: \mathrm{Zn}$ low voltage phosphors research, the applied voltage was about $300 \mathrm{~V}$ [19]. Thus, it does not yet meet the requirement for low voltage flat panel display usage. 


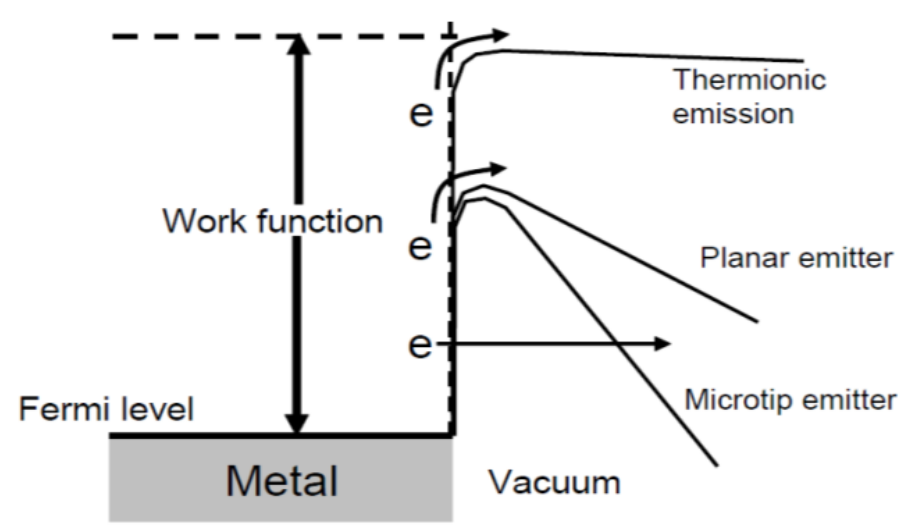

Figure 1. Schematic diagram of the field emission barriers for a planar and a micro-tip emitter.

\section{Organic light emitting diodes (OLEDs)}

Using organic material for light emitting diodes (LEDs) is fascinating due to their vast variety and relative ease of controlling their composition to tune their properties by chemical means. For example, by applying an electric field to an anthrancene single crystal, Pope et al. in 1965 observed blue electroluminescence (EL) [20]. Soon after alternating current EL was also achieved using an emissive polymer [21].The observation of efficient bright EL, defined as the number of photons emitted from the face of the device per injected electron or hole, the investigation on the organic optoelectronic device commenced to investigate and developed slowly until Tang and Vanslyke demonstrated efficient green electroluminescence [22] from a vapor deposited organic compound in 1987. Till now, OLEDs are the best flat light emitting source.

\subsection{Structure of organic light emitting diodes (OLEDs)}

A typical OLED is composed of an emissive layer, a conductive layer, a substrate, and both anode and cathode terminals. The basic structure of a typical OLED is shown in Fig. 2. The first layer above the glass substrate is a transparent conducting anode, typically indium tin oxide (ITO). In Flexible OLEDs the anode is made of a transparent conductance plastic substrate. There are two different types of OLEDs. Traditional OLEDs use small organic molecules deposited on glass to produce light. The other type of OLEDs uses large plastic molecules called polymers. The single or multilayer small organic molecular or polymer film is deposited on the transparent anode. Appropriate multilayer structures are used to enhance the performance of the device by lowering the barrier for hole injection from the anode and by controlling the electron and hole recombination region. The injected holes move from the interface of the organic/electrode into the organic light emitting layer, where the defect density is high. Therefore, the organic layer deposited on the anode should generally be a good hole transport layer (HTL). Similarly, the organic layer in contact with the cathode should be an optimized electron transporting layer (ETL). Generally, the anode 
of OLEDs is an ITO film, the cathode is typically a low-to-medium work function $(\Phi)$ metal such as $\mathrm{Ca}(\Phi=2.87 \mathrm{eV}), \mathrm{Al}(\Phi=4.3 \mathrm{eV})$ or $\mathrm{Mg}_{0.9} \mathrm{Ag}_{0.1}(\mathrm{Mg}, \Phi=3.66 \mathrm{eV})$ deposited either by e-beam or thermal evaporation [23].

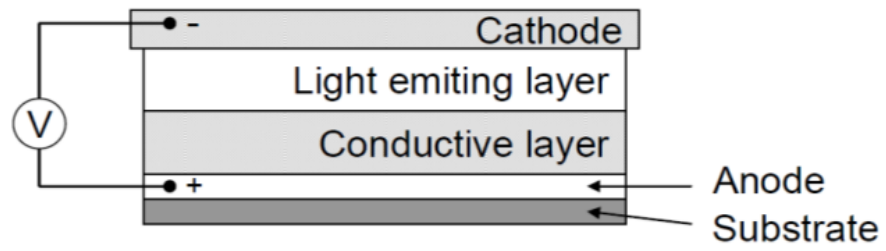

Figure 2. Schematic structure of organic light emitting diodes.

\subsection{Mechanism of the operation of organic light emitting diodes (OLEDs)}

The light emission from OLEDs is through electroluminescence (EL), which can be described in three steps (see Fig. 3) as follows: step 1: when a forward bias voltage is applied to an OLED, holes and electrons are injected. These injected charge carriers have to overcome their respective interface barriers and then holes occupy into the highest occupied molecular orbital (HOMO) energy level of the hole transport layer (HTL) and electrons into the lowest unoccupied molecular orbital (LUMO) energy level of the electron transporting layer (ETL). The HOMO of HTL is similar to the valence band in bulk semiconductors, and LUMO of ETL is similar to the conduction band. Step 2: The externally applied field on OLED drives the injected holes and electrons to the interface of HTL and ETL, where they are accumulated; holes in HOMO of HTL and electrons in LUMO of ETL. Step 3: Due to organic solids have low dielectric constant and strong binding energy both carriers ( holes and electrons) move toward the interface between the two transport layers (HTL and ETL) and recombine in the light emitting layer (EML) to form excitons. Then these excitons emit light through the transparent electrode (ITO coated on glass substrate). In general environment, the exctions exist in an unstable state and their radiative move to recombination releases energy in the form of the light and heat. According to the above three steps, illustrated in Fig. 3, the light emission from an OLED is current driven and hence called electroluminescence (EL).

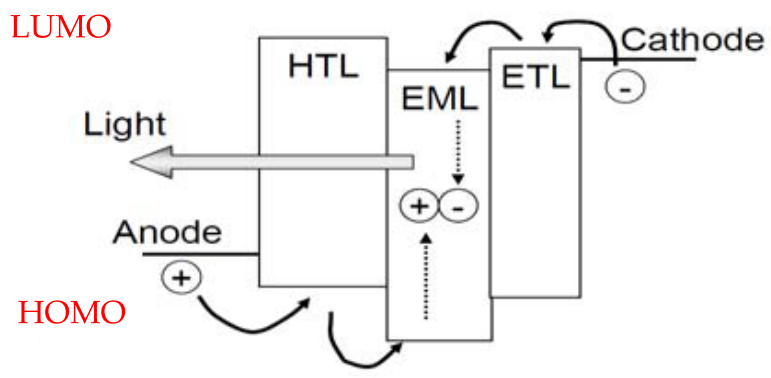

Figure 3. Schematic sketch of the active energy levels of organic light emitting diodes (OLEDs) 
The carrier transmission in organic molecules is different from that in inorganic semiconductors or crystalline materials. There are no continuous energy bands in organic semiconductors. In organic semiconductors, consisting of organic molecules, there are delocalized $\pi$ electrons, which are relatively free but confined on individual molecules due to weak intermolecular interactions. Therefore, the hopping theory is the most commonly used to describe the phenomenon of carrier transfer in organic solids. Driven by the electric field, electrons are excited or injected into the LUMO of one molecule in ETL and hop to the LUMO of neigh-boring molecule and thus electron transport occurs. Likewise, injected holes get transported by hopping from the HOMO of one molecule to another in HTL. In fact, as the charge carriers are injected externally and do not exist before the application of the electric field, the location of holes in HOMO and electrons in LUMO deforms the associated bond length and structure. Therefore, the movement of an injected electron or hole is coupled with the local deformation zone to form a unit, this unit is called polaron. Hence, in organic semiconductors the movement of the electrons or holes is often accompanied by the deformation of its structure, which is called self-trapped electron or hole. As such selftrapped charge carriers move slower the carrier mobility in organic semiconductors is in general lower than that in inorganic semiconductors or metals. The hole mobility in organic materials is typically $10^{-7}-10^{-3} \mathrm{~cm}^{2} / \mathrm{Vs}$ and the electron mobility is typically lower by a factor of 10-100 [24].

The organic materials are usually insulators (such as plastic). Generally only a very small amount of current can be injected into the organic material by applying certain electric field, and EL occurs from the recombination of these injected electrons and holes. Therefore, if the current is less then injected carriers will be less and number of excitons to recombine will be limited. Therefore, to a large extent EL depends on improving the carrier injection efficiency from both electrodes, and on obtaining balanced and controlled electron-hole recombination within a well-defined zone.

\subsection{Full Color of organic light emitting diodes (OLEDs)}

There are five potential methods to make an OLED emit in red (R), green (G) and blue (B) color spectral regions [35]: 1). side by side patterning of red (R), blue (B) and green (G) OLED's, 2). absorptive filtering of white OLED, 3). fluorescent down-conversion of blue OLED's, 4). microcavity filtered OLED's and 5). color-tunable OLED's.

Method (1) This method is employed a precisely positioned shadow mask to selectively deposit the R $、 G$ and B OLEDs with individual pixels of $R \cdot G$ and B emission.

Method (2) A white OLED device can be made using materials with very broad emission spectra, or using two or more sequentially deposited light emitting layers and then color filters on white light OLEDs are used to change the emission into R, G and B colors.

Method (3) The full color pixel can be using a single blue OLED to pump wavelength downconverters, which efficiently absorb blue light and re-emit the energy as green or red light. 
Method (4) The emission from a white OLED is filtered by a microcavity, which is composed of a dielectric quarter wavelength stack as the bottom mirror, the metal contact as the top mirror and an inactive material as a filler layer to adjust the cavity thickness [26]. However, the microcavity resonance causes strong viewing angle dependence of emitted colors, limiting this method to applications which need small viewing angle. In this method about $\pm 15^{0}$ viewing angle can be achieved [27].

Method (5) The color variation is achieved by voltage and/or polarity tuning. Only molecular OLEDs are capable of three color tuning. This method shows low efficiency and/ or requires high driving voltage. Hence, the color variable devices based on the polarity and/ or voltage-tuning are still far from applications. White light emission OLEDs can also serve as backlight panels of LCDs. White is the most important color in the lighting industry. A number of device structure concepts have been proposed to achieve white emission. These include the mixing of three primary colors from respective layers in a multilayer structure [28], the doping of appropriate amount of red, green, and blue dopants in the same host [29], the microcavity effect of one emission layer [30], use of exciplex formation, etc.

OLEDs have become viable now for flat panel displays after intensive research and progress in the past decade. Through proper material design/choice and device fabrication, various OLEDs with colors of high brightness have been developed for use in single- or full-color applications. As the operation of an OLED depends on the carrier transport in HTL and ETL, hole and electron confinement in EML and then their recombination to emission light. In most cases the number of injected holes in an OLED is more than electrons. Therefore, improving efficient electron injection is essential for efficient and stable OLEDs.

\section{The field emission organic light emitting diodes (FEOLEDs)}

\subsection{Structure of FEOLEDs}

As shown in Fig. 4, the basic structure of a FEOLED is to utilize an organic EL light-emitting material instead of inorganic phosphor thin film in FEDs [31]. The anode of the FEOLEDs can be a multi-layered organic solid or an OLED . But both have the same structure, which includes a hole injection layer (HIL), a hole transport layer (HTL) and a light emitting layer (EML). The cathode of FEOLEDs is made of CNTs template as electron source. In such a structure, it is not only difficult to protect the light emitting layer (EML) from high-energy electron bombardment [32], but also not easy to control highly efficient emission.

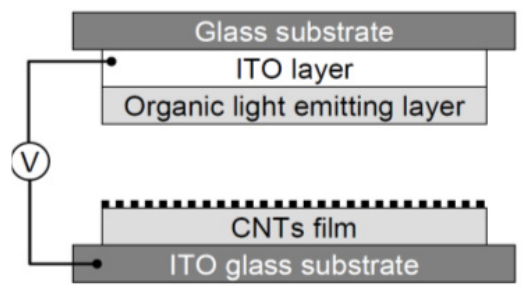

Figure 4. The schematic diagram of a FEOLED. 
Since the basic structure of a FEOLED uses a direct current (DC), a protection layer is needed. Additionally, it can be deposited on EML. Such a protection layer in FEOLEDs increases the operating lifetime since this layer protects against electron bombardment .To improve this, a few different structures of FEOLEDs are presented in Figs.5. and 6.

Fig. 5 (a) shows the device structure of a FEOLED with the protection layer, which is made of a secondary electron material used as an electron multiplier. By apply operating voltage to FEOLEDs, the electron and hole recombination occurs in the EML by mechanisms similar to OLEDs. As OLEDs are current injection devices, electron injection must be improved to ensure their efficiency and stability. Therefore, as shown in Fig. 5(b), a field emission electrons layer is introduced in OLEDs to increase the electron density, which exhibits a higher luminous efficiency in FEOLEDs than conventional OLEDs [33]

Fig.6 shows the schematic diagrams of FEOLEDs with a dynode in (6a) and a strip electron multiplier in $6(\mathrm{~b})$. According to the above described mechanism of FEOLEDs, the luminance intensity increases with the increase in electron injection. A common way to solve this problem is to introduce a dynode or an electron multiplier into the FEOLEDs . As shown in Fig.6 (a), dynode has holes whose whole inner surface is coated with a secondary electron material such as $\mathrm{Be}, \mathrm{Mg}$, or $\mathrm{Ca}$ oxide to increase the electron amplification factors. Fig.6 (b) shows a FEOLED device with strip electron multiplier. The strip electron multiplier was first proposed here by the author, which consisted of a strip of Al coated with another striped secondary electron material [3]. Both of the dynode and strip electron multiplier used in FEOLEDs are made for increasing the number of electrons and allow carriers to achieve a more balanced state in OLEDs and then enhance the luminance efficiency of OLEDs.

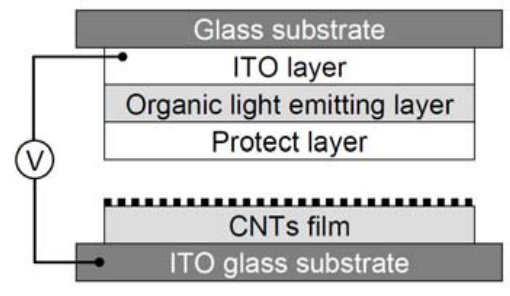

(a)

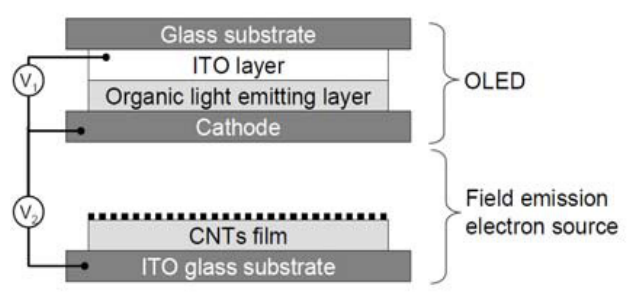

(b)

Figure 5. Two configurations of FEOLEDs (a) with protection layer and (b).with Al metal cathode. 


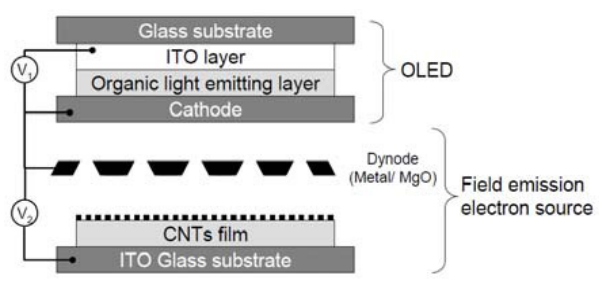

(a)

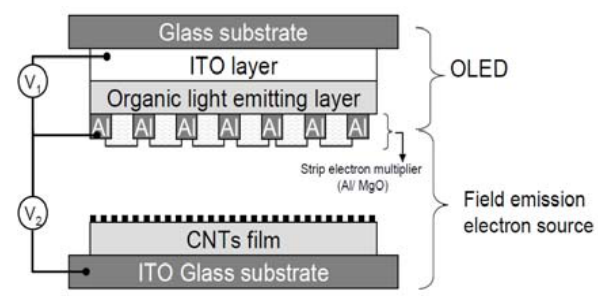

(b)

Figure 6. Two configurations of FEOLEDs with (a).Dynode and (b).strip electron multiplier

\subsection{Fabrication of FEOLEDs}

A FEOLED is that an organic emission layer (organic EL) is utilized instead of inorganic phosphor thin film in field emission display (FED). The organic EL in a FEOLED consists of a hole injection layer (HIL), a hole transport layer (HTL) and light emitting layer (EML). A FEOLED is able to attain higher luminance and low power consumption than conventional OLED.

Fig.7 (a) shows the structure of a basic FEOLED. The anode of FEOLED is ITO on which are coated the organic multi layers. The cathode consists of a field emission electron, which provides the electron injection into the organic material layer. Fig.7 (b) shows the FEOLED structure with a dynode, which acts as an electron multiplier to increase the number of electrons injected into the organic layer.

Fig. 8 (a) shows the structure of FEOLED with the strip electron multiplier. Due to the dynode is difficult to set in the narrow space of FEOLEDs. Therefore, the strip electron multiplier was to be proposed. Refer to Fig. 7(b), the dynode can be of the metal channel, box, line focus, or MPC type [1]. The secondary electron material in the dynode can be $\mathrm{Cu}$ Be or Ag-Mg alloys. However, the strip electron multiplier was form with the secondary electron material (MgO or CsI) and $\mathrm{Al}$, as shown in Fig. 8 (b).

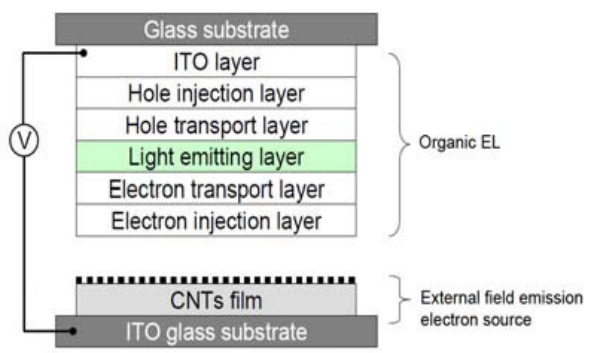

(a)

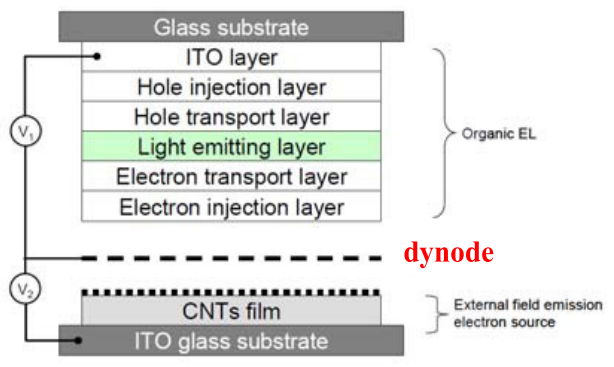

(b)

Figure 7. The components of FEOLED with (a) the basic device and (b) dynode structure. 
Fig. 8 (c) shows the operating principle of a dynode, which is a cross-sectional schematic view of the metal channel type dynode. The secondary electron emission generated from the dynode can be understood by the following processes: (a) the primary electrons penetrate into a certain depth of an insulating layer (the secondary electron material); (b) through collision the energy of primary electrons is transferred to bound electrons of the insulator to release them and (c) released electrons migrate to the surface and escape into the vacuum as secondary electrons.

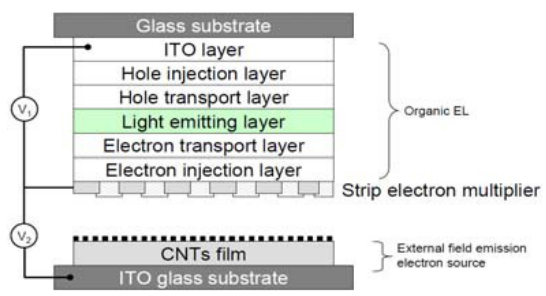

(a)

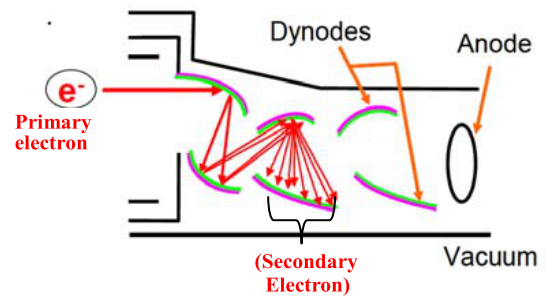

(c)

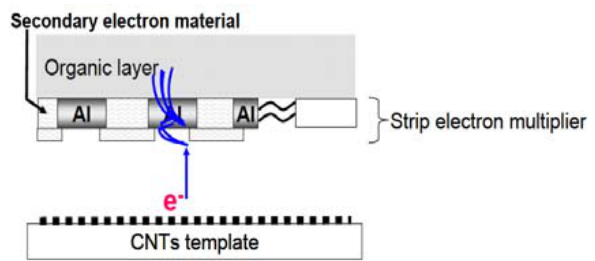

(b)

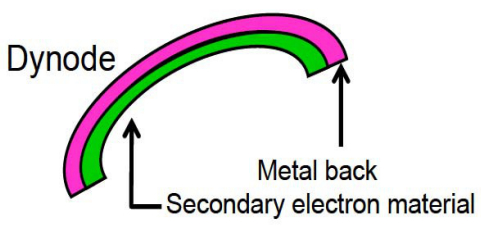

(d)

Figure 8. Schematic structure of a FEOLED with (a) strip electron multiplier and (b) strip Al. (c) the operating principle of a dynode and (d) A sheet of a dynode

\subsection{Organic light emitting diode in FEOLED}

In Fig. 8 (a) is shown a FEOLED with the strip electron multiplier formed on an OLED as a part of anode. The organic multilayer structure of FEOLED is the same as that of OLED, which works with the same mechanism.

One of the most important issues related with the characteristics of OLEDs is the number of injected electrons and holes should be balanced. It is well know that the direct electron-hole recombination in the light emitting layer occurs due to OLEDs. Therefore, an effective cathode structure for efficient electron injection is critical to optimal performances of OLEDs. A nanometer-size interfacial layer between the metal cathode and organic material in OLEDs plays the critical role in the carrier injection efficiency. In order to improve the injection efficiency of electrons, the low work function metal or alloys such as LiF are usually used to form low energy barriers for electron injection from the cathode to the 
organic material [34]. It has been shown that LiF is very effective in terms of facilitating electron injection. However, recently alkali metal carbonates $\left(\mathrm{Cs}_{2} \mathrm{CO}_{3}\right)$ have also been reported to be efficient electron injection material [35, 44-45]. We believe that if the electrons are efficiently injected from the cathode to organic layer, then this will improve the charge carrier balance in OLEDs and hence improve the device efficiency. This idea will work the same way in FEOLEDs.

\subsection{Carbon nanotubes field emission template in FEOLEDs}

In this section, FEOLEDs are discussed for their optoelectronic characteristics in terms of external electron supplement into the organic light emitting layer. Hence, FEOLEDs must have an excellent field emission cathode to emit electrons. The carbon nanotubes (CNTs) template is the best choice to be adopted in FEOLEDs as the field emission cathode. CNTs can work in less stringent vacuum conditions $\left(<1 \times 10^{-5}\right.$ torr $)$ and have higher emission currents than metal and semiconductor micro-tip field-emission sources. Iijima discovered carbon nanotubes (CNTs) in 1991[36]. CNTs have a superior mechanical strength, good heat conductance, and ability to emit cold electrons at relatively low voltages because of their high aspect ratios and nanometer-scale tips.

The traditional method of fabricating field emitters is based on the use of multi-needle field emission cathodes and precision technological processes based on electron lithography techniques. Metal and semiconductors are usually used as cathode materials, which, unfortunately, have rather high work functions $(4-5 \mathrm{eV})$ [37]. The application of CNTs in field emission template is very extensive. For example, CNTs can be directly synthesized on a substrate by CVD on an anodic aluminum oxide (AAO) template, and by screen print. However, the fabrication of a CNTs template is time consuming. There is a new and more convenient method to fabricate the CNTs templates by the spray method [38-39].

CNTs thin films are usually fabricated by two methods, such as: drop-drying from solvent [41] and filtration and spin-coating [42], but these methods have severe limits in the film quality, like in uniformity, homogeneity, and production efficiency. CNTs thin films consisting of multi-walled CNTs (MWCNTs) are fabricated by the spray method, which is an easy and convenient method to deposit CNTs and can achieve large area deposition [43]. The procedures of the fabrication of CNTs template are described as below: First CNTs are suspended in 1, 2- dichloroethylene (DCE) and second, sonication of $30 \mathrm{mg}$ CNTs in $50 \mathrm{ml}$ DCE solvent for 2 hours. To obtain good adhesion between CNTs and ITO glass substrate, an indium (In) metal layer is deposited onto the ITO glass substrate by thermal evaporation. After annealing at $300{ }^{\circ} \mathrm{C}$ for $15 \mathrm{~min}$ in N2 atmosphere, CNTs are firmly adhered to the In layer and produce good field emission characteristics.

\subsection{Operation principle of FEOLEDs}

According to the above mentioned FEOLED structure, FEOLEDs can be divided into two models: 1) Original type FEOLEDs and 2) triode devices. 


\subsubsection{Original type FEOLEDS}

As shown in Fig. 7 (a), FEOLEDs (original type) are similar to FEDs, but they use organic light emitting material instead of a phosphor. In these FEOLEDs an ITO film is used as anode and CNTs template as cathode. By applying the driving voltage to both the electrodes (ITO and CNTs template), then electrons and holes move toward the interface between the two transport layers (HTL, and ETL) and recombine to form excitons. Finally, these excitons emit light through the ITO substrate..

\subsubsection{Triode FEOLEDs with a dynode structure (strip electron multiplier)}

As shown in Fig, 7 (b), FEOLEDs with a dynode structure are classified as triode devices. It comprises the dynode and an organic EL light emitting layer and CNTs template. The dynode is formed between the cathode and the organic EL lighting layers.

As shown in Fig. 8 (a), there is another kind of triode FEOLED, which is a FEOLED with a strip electron multiplier. In this case a strip electron multiplier is used instead of a dynode, but it is attached directly to the organic EL light emitting layer for protecting the electron injection layer from high-energy electron bombardment and allows the electrons to disperse evenly in the EML.

\subsubsection{Luminescence mechanisms in FEOLEDs}

To further confirm that the mechanism of operation of FEOLEDs is the same as that of OLEDs, the following experiment was conducted. A hole blocking layer (BCP) is inserted between the hole transport layer (NPB) and the emission layer ( $\left.\mathrm{Alq}_{3}\right)$ of the organic formation, as shown in Fig. 9(a). If an OLED is applied a voltage, hole carriers injected from the ITO anode electrode to the hole transport layer would be blocked at the interface of the NPB layer and the BCP layer. The electrons (emitted from the cathode and passing through Alq3) would then recombine with the holes accumulated in the NPB layer. Refer to Fig. 3, this NPB excitation generated by the recombination, according to the active energy levels of OLEDs would give rise to blue light. The Alq3 layer in such case would generate no light. If a cathode luminescence mechanism device is applied with the BCP layer, on the other hand, electron bombardment on the organic material would generate light in the emission layer $\mathrm{Alq}_{3}$, which should have green color. As such, when a BCP layer is inserted in FEOLEDs, if blue light is observed, the luminescent mechanism of the FEOLED must be similar to that of the conventional OLED: if green light is observed, the luminescent mechanism must be similar to that of cathode luminescence. The experiment results showed that blue light was observed, as shown in Fig. 9(b), which clearly illustrates that both FEOLEDs and OLEDs operate on similar mechanism of emission.

Thus, the light emission in FEOLEDs also occurs via the following five processes as in OLEDs: 1) both electrons and holes injected from anode and cathode into organic layers, 2) these injected charge carriers are transported towards each other across the organic layer, 3) formation of singlet excitons due to the Coulomb interaction between the injected electrons 
and holes, 4) migration singlet excitons to organic emitting layer and 5) radiative recombination of single excitons.

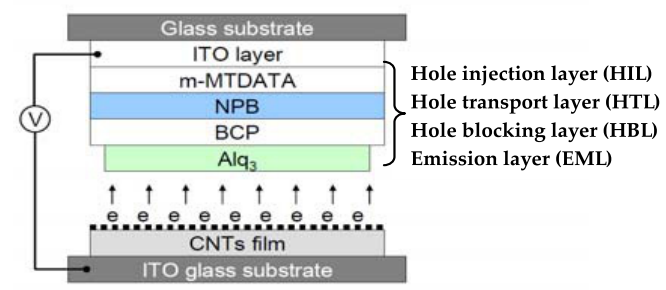

(a)

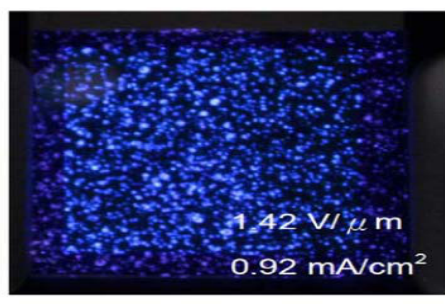

(b)

Figure 9. (a) Structure of a FEOLED with Hole blocking layer and (b) emission of blue light.

\subsection{A FEOLED with strip electron multiplier}

As shown in Fig. 10, the typical structure of FEOLEDs in this work comprises a CNTs template cathode and the strip electron multiplier formed on an OLED as a part of anode. It can be assembled in a vacuum chamber.

\subsubsection{Experimental}

In this section, the current density $(\mathrm{J})$-applied voltage $(\mathrm{V})$ characteristics and the optical performances of a FEOLED with the strip electron multiplier are studied experimentally.

Fig.10 shows the configuration of FEOLED in this work, where the upper portion is an OLED lower part is the external electron source of CNTs cathode. The Soled switch is use to control

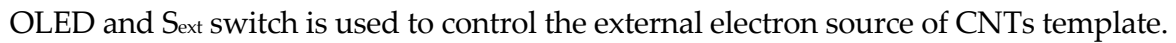

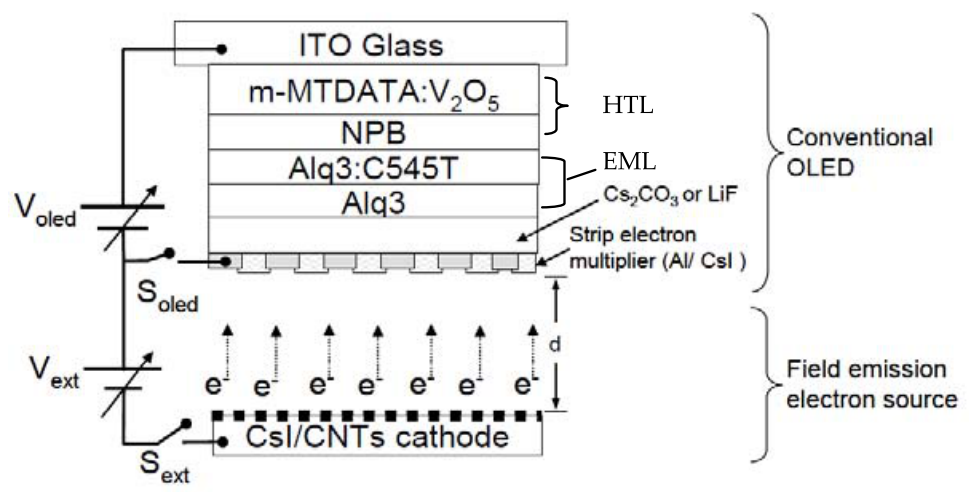

Figure 10. Schematic presentation of an apparatus for characterizing a FEOLED with strip electron multiplier 
The structure of an OLED is ITO glass/ m-MTDATA: $\mathrm{V}_{2} \mathrm{O}_{5}(10 \mathrm{~nm}, 10 \%) / \mathrm{NPB}(30 \mathrm{~nm}) /$ Alq3:C545T (30nm, $3 \%) /$ Alq3 $(10 \mathrm{~nm}) / \mathrm{Cs}_{2} \mathrm{CO}_{3}(1 \mathrm{~nm}) / \mathrm{Al}$, where, the $\mathrm{V}_{2} \mathrm{O}_{5}$ doped mMTDATA was chosen as the hole transport layer (HTL) and it has a high conductance, intrinsically leading to the formation of many intrinsic carriers between ITO and the organic surface significantly enhancing the hole injection and transport [46]. Additionally, the emission layer (EML) of OLED uses Alq3 as host and C545T dye as green fluorescent material to trap the electrons to build up the space charge and decrease the free electron distribution in the host Alq3 [47], subsequently reducing the current density of the device. Moreover, $\mathrm{Cs}_{2} \mathrm{CO}_{3}$ and $\mathrm{LiF}$ were chosen as electron injection buffer layer (EIL). And the strip electron multiplier was fabricated from the strip of $\mathrm{Al}(80 \mathrm{~nm})$ and strip of CSI $(110 \mathrm{~nm})$ and integrated with the organic material of FEOLEDs. The entire organic material layer was prepared by using a high vacuum thermal evaporation system.

Such a structure (Fig.10) is used to examine the current-voltage (J-V) characteristics of OLEDs with a different electron injection layer to prove the electron injection capability of $\mathrm{Cs}_{2} \mathrm{CO}_{3}$. Additionally, the J-V characteristics and luminance of the FEOLED are measured by Keithly-2400, Keithly-237 and TOPCON PR-650, respectively. All the measurements are performed in a high vacuum ambient of $6 \times 10^{-6}$ torr at room temperature.

\subsubsection{Results and discussion}

In this section, the current density (J)-applied voltage $(\mathrm{V})$ characteristics and the optical performances of a FEOLED with Electron Multiplier are studied experimentally.

Fig.11 (a) shows the J-V characteristics of two OLEDs, one with LiF and the other with $\mathrm{CS}_{2} \mathrm{CO}_{3}$ as the electron injection layer when Soled is closed and Sext is open ( Fig10). To enhance the OLED performance, the $\mathrm{CS}_{2} \mathrm{CO}_{3}$ is used instead of $\mathrm{LiF}$ as the electron injection material. The optimal thickness of $\mathrm{CS}_{2} \mathrm{CO}_{3}$ has been characterized and found to be $1 \mathrm{~nm}$. Under the same driving voltage of $10 \mathrm{~V}$, the OLED with the electron injection layer of $\mathrm{CS}_{2} \mathrm{CO}_{3}(1.0 \mathrm{~nm})$ can get the current density of $93 \mathrm{~mA} / \mathrm{cm}^{2}$ which was higher than that of OLED with the electron injection layer of $\operatorname{LiF}(0.7 \mathrm{~nm})$. The better performance of OLEDs with $\mathrm{Cs}_{2} \mathrm{CO}_{3}$ can be attributed to $\mathrm{Cs}$ having a low-work function of $2.14 \mathrm{eV}$ relative to $\mathrm{Li}$ (2.9 eV) [50]. The electron injection layer of $\mathrm{Cs}_{2} \mathrm{CO}_{3}$ in OLEDs seems to have induced strong ndoping effects in Alq3 and ultimately increases the electron concentrations in the electronstransport layer of Alq3. Moreover, OLED with an increased thickness of $\mathrm{Cs}_{2} \mathrm{CO}_{3}$ to $2 \mathrm{~nm}$ have shift the $\mathrm{J}-\mathrm{V}$ curve to a lower current density. Notably, the OLEDs performance depends on the thickness of the $\mathrm{Cs}_{2} \mathrm{CO}_{3}$ layer.

Fig.11 (b) shows the luminance (L)-applied voltage (V) characteristics of the same two OLEDs with $\mathrm{LiF}$ and $\mathrm{CS}_{2} \mathrm{CO}_{3}$ as the electron injection layer when Soled is closed and $\mathrm{Sext}_{\text {is }}$ open ( Fig10). According to Figure (b), the OLED with $\mathrm{Cs}_{2} \mathrm{CO}_{3}(1 \mathrm{~nm})$ as the electron injection layer can achieve a high luminance of $10,820 \mathrm{~cd} / \mathrm{m}^{2}$ and a high EL efficiency of 12 $\mathrm{cd} / \mathrm{A}$ at $10 \mathrm{~V}$. In contrast, OLED with a LiF layer can achieve a luminance of $5,821 \mathrm{~cd} / \mathrm{m}^{2}$ and 
an EL efficiency of $10.2 \mathrm{~cd} / \mathrm{A}$ at $10 \mathrm{~V}$. This demonstrates very clearly that the OLEDs, in which $\mathrm{CS}_{2} \mathrm{CO}_{3}$ is used as an electron injection layer, show an excellent performance. It indicates that electrons are effectively injected from the cathode to the organic layer due to the lower electron injection barrier, which improves the charge carrier balance and subsequently increases the device efficiency.

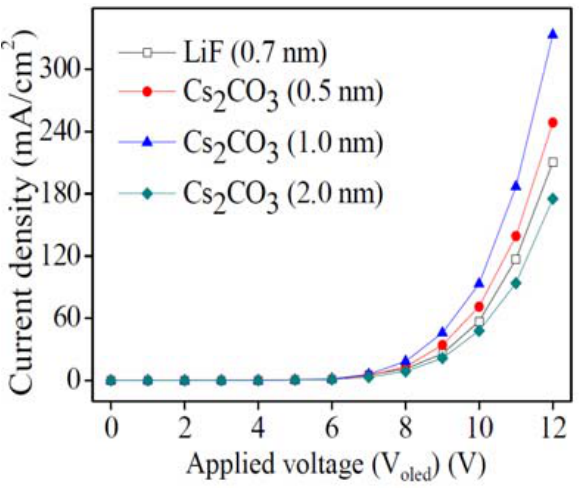

(a)

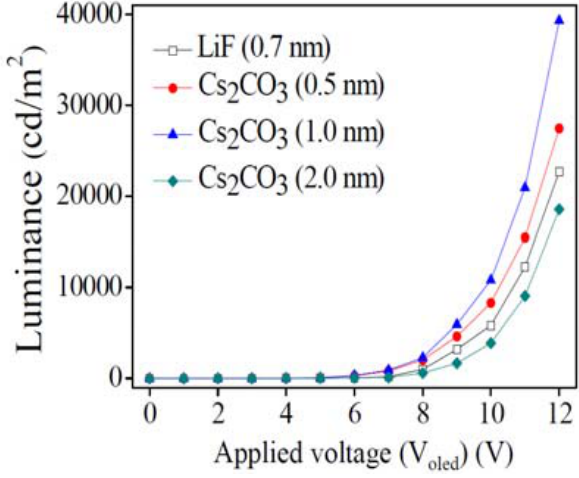

(b)

Figure 11. (a) Current density (J)-Applied voltage (V) at SOLED closes and Sext is open and (b) Luminance $(\mathrm{L})$-Applied voltage $(\mathrm{V})$ at Soled=closes and Sext is open(Fig.10)

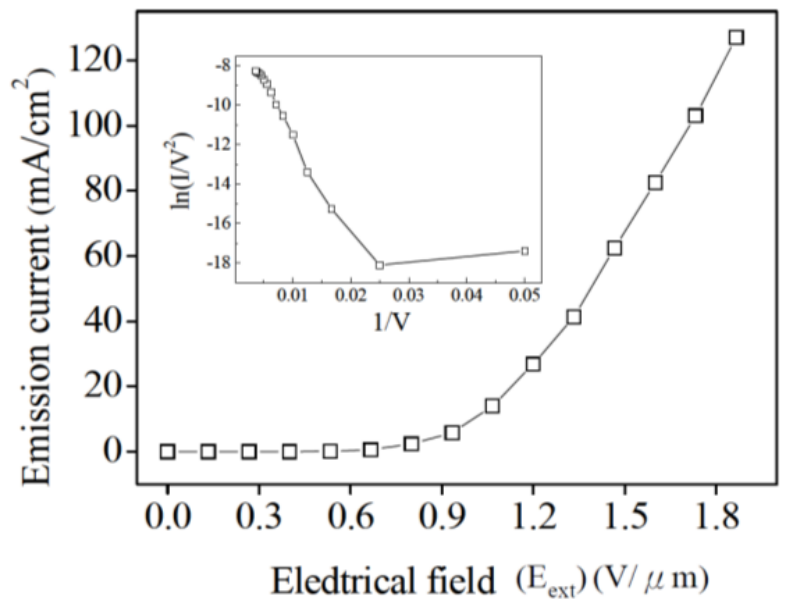

Figure 12. The field emission current versus electric field (J-E) characteristics of a CNTs template when Soled is open and Sext is closed, (Eext=Vext/d) (Fig. 10)

Fig. 12 shows the field emission current versus electric field (J-E) characteristics of a CNTs template. The CNTs template is made as an external electron source for FEOLED [51]. A field emission current density of approximately $127 \mathrm{~mA} / \mathrm{cm}^{2}$ is produced at an electric field of $1.86 \mathrm{~V} / \mu \mathrm{m}$. The enhanced current density can be attributed to the satisfactory adhesion 
between CNTs and the ITO glass substrate. The current density increases with the electric field. Based on the above results, the amount of electrons injected into the Al electrode can be determined by adjusting the electrical field (Fig.10, Vext), which is applied to the CNTs template. Detailed operations of FEOLEDs can be described as follows. Initially, Soled is turned on to drive the OLED shown in Fig.10. The OLED emits a luminance of $10,820 \mathrm{~cd} / \mathrm{m}^{2}$ as the driving voltage reaches $10 \mathrm{~V}$ and, simultaneously, Sext is switched on to attract the electrons emitted from the CNT emitters.

Fig.13 shows the luminance-current density characteristics of OLEDs and FEOLEDs. The curve in section A displays the characteristics of a conventional OLED (Soled=close, $\mathrm{S}_{\text {ext }}=$ open, as shown in Fig.10), where sections B and C show the FEOLED (Soled=close, $\mathrm{S}_{\mathrm{ext}}=\mathrm{close}$, as shown in Fig.10). At a driving voltage of $10 \mathrm{~V}$ on an OLED, the luminance is enhanced from $10,820 \mathrm{~cd} / \mathrm{m}^{2}$ to $27,393 \mathrm{~cd} / \mathrm{m}^{2}$ while Sext is turned on. Obviously, applying an electrical field (Eext) to the CNTs template can enhance the generation of the field emission electrons into the OLED. Additionally, the current density of OLED is increased by the supplementary electrons into the multilayer of the organic light emitting layer with the external electron source (Soled=close, $S_{\text {ext }}=$ close, as shown in Fig.10). Moreover, the current density of the OLED $\left(\mathrm{V}_{\text {oled }}=10 \mathrm{~V}\right)$ with the external electron source increases from $93 \mathrm{~mA} / \mathrm{cm}^{2}\left(\mathrm{E}_{\text {ext }}=0.8\right.$ $\mathrm{V} / \mu \mathrm{m})$ to $184.5 \mathrm{~mA} / \mathrm{cm}^{2}\left(E_{\text {ext }}=1.7 \mathrm{~V} / \mu \mathrm{m}\right)$, and the luminance also increases from $10,820 \mathrm{~cd} / \mathrm{m}^{2}$ to $27,393 \mathrm{~cd} / \mathrm{m}^{2}$ simultaneously, as shown in Fig.13 (hole block line)

According to the above characteristics of FEOLED in comparison with the OLED under the same operating current density $\left(120 \mathrm{~mA} / \mathrm{cm}^{2}\right)$, the FEOLED exhibits a higher luminous efficiency of $18.6 \mathrm{~cd} / \mathrm{A}$ than the luminous efficiency of $11.42 \mathrm{~cd} / \mathrm{A}$ for OLED, as shown in Fig.14. The FEOLED results can be attributed to the external electron injection into the multilayer organic layer of OLED, thus balancing the hole and electron. Furthermore, increasing the quantity of electrons by using an external electron source significantly increases the current density of OLED and makes the luminance efficiency higher than that of conventional OLED.
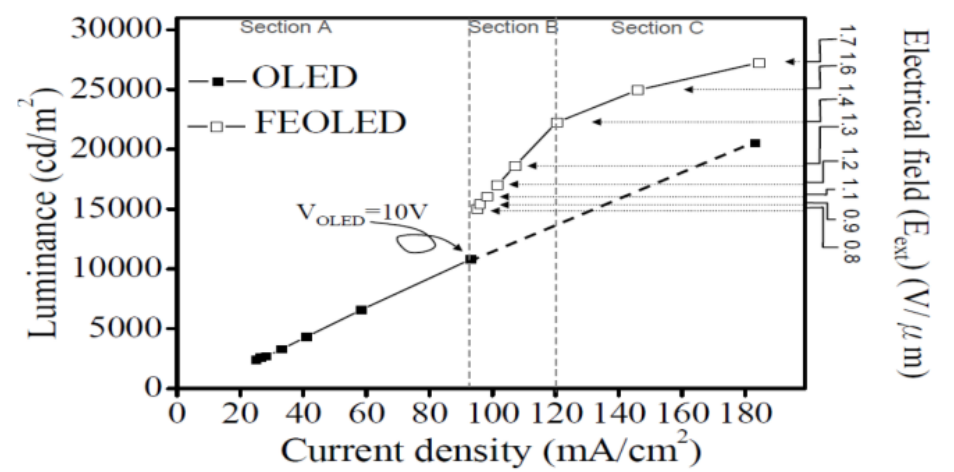

Figure 13. The Luminance (L)-Current density (J)-Electric field (E) of the OLED and FEOLED devices at both Soled and Sext close (Eext=Vext/d) (Fig. 10) 


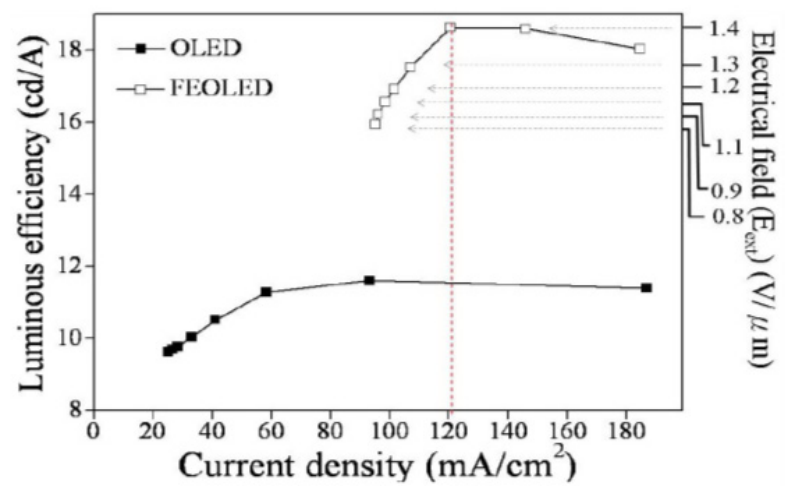

Figure 14. The Luminous efficiency $(\eta)$ - Current density (J)-Electrical field (E) of the OLED and FEOLED devices at both Soled and Sext close (Eext=Vext/d)

In a FEOLED, the electrical field under vacuum condition, accelerates the electrons emitted from the CNTs cathode to affect the secondary electron material of CsI; they then pass through the $\mathrm{Al}$ and transport are transported through the organic EL light emitting layer. Field emission electrons with a sufficiently large electron energy supplement into OLED to increase the current density. Notably, increasing the number of the electrons that reach the organic EL light emitting layer also increases the luminous efficiency of the OLED. Therefore, the ways in which the OLED and FEOLED differ can be easily observed under the same current density. The luminance of FEOLED exceeds that of conventional OLED, as shown in Fig.13. Our results further demonstrate that the curve of the FEOLED becomes gradually saturated, especially for section C. Notably, injecting external electrons into the OLED continuously does not allow the luminance of the FEOLED to increase linearly with the current density since the quantity of electrons is larger than in the hole in section $\mathrm{C}$. The carrier has become imbalanced again, subsequently decreasing the luminance. Furthermore, the electronic behavior shown in the FEOLED, it can be further demonstrates the amount of electrons is less than holes. 
As describe above, we can see that the characteristics of the OLED and the FEOLED are listed by Table 1, respectively.

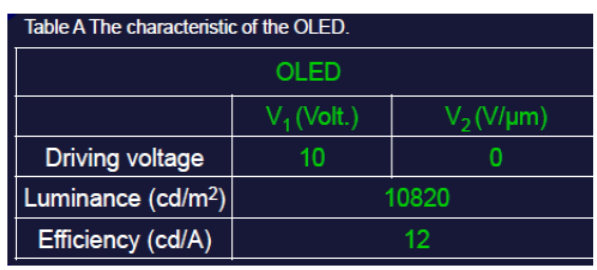

(1)

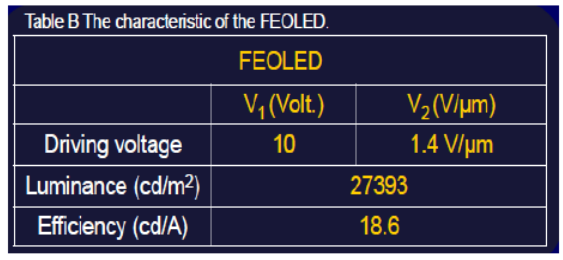

(2)

Table 1. (1) The characteristics of the OLED and (2) The characteristics of the FEOLED

\section{Conclusion and future direction}

This work presents a novel FEOLED had further increase the luminous efficiency of OLEDs. The characteristics of an OLED constructed in the FEOLED device are optimized by inserting a thin $\mathrm{Cs}_{2} \mathrm{CO}_{3}$ electron injection layer between the $\mathrm{Alq}_{3}$ and $\mathrm{Al}$ electrode. Experimental results indicate that the external field emission electrons can enhance the luminance in FEOLED efficiently owing to balanced recombination of electrons and holes. Additionally, FEOLED achieves a higher luminous capability than that of OLED under the same current density. Mechanism detection of the FEOLED further reveals that the amounts of holes are more than that of electrons in the emission layer of an OLED device. Furthermore, the secondary electron material CsI deposited onto the Al electrode in a FEOLED can provide multiple electrons as well as prevent the organic layer from electrons bombardment. The proposed device's construction is extremely important for characterizing the emission mechanism of the FEOLED.

Another objective of this chapter is to provide background knowledge to readers from the different fields to stimulate new ideas. For example, the flexible photovoltaic OLED (PVOLED)

and a tandem of organic solar cell (OSC) and white organic light emitting diode (WOLED), although not addressed here, are now emerging. In PVOLEDs, the power recycling efficiency of $10.133 \%$ is achieved under the OLED of PVOLED operated at $9 \mathrm{~V}$ and at a brightness of $2110 \mathrm{~cd} / \mathrm{m}^{2}$, when the conversion efficiency of OSC is $2.3 \%$.[52]. In a tandem of OSC and WOLED, which can be fabricated to generate electricity as well as lighting for domestic and commercial uses [53]. 


\section{Author details}

Meiso Yokoyama

Department of Electronic Engineering, I-Shou University, Kaohsiung City, Taiwan

\section{References}

[1] Meiso Yokoyama, U.S. Pat. 7,456,562 B2, (2008)

[2] Meiso Yokoyama, Japan Patent 3879101, (2006)

[3] Meiso Yokoyama, Chi-Shing Li, and Shui-Hsiang Su,"Novel Field Emission Organic Light Emitting Diodes" IEICE TRANS. ELECTRON.,VOL. E94-C,NO.3, (2011)

[4] Chi-Shing Li, Meiso Yokoyama, and Shui-Hsiang Su, "Efficiency Enhancement of FieldEmission Organic Light Emitting Diodes Using a Dynode Structure" Electrochemical and Solid-State Letters, vol. 11, pp. J1-J3,(2008)

[5] Meiso Yokoyama et al. The $6^{\text {tsh }}$ internal Symposium on Organic Molecular Electronics (ISOME 2010),( 2010)

[6] G. T. Chen, S. H. Su, and M. Yokoyama, "Field-Emission Organic Light-Emitting Device Using Oxide-Coated Cathode as Electron Source" Electrochem. Solid State Lett., vol. 10, no. 3, pp. J41-J44,( 2006)

[7] G. N. Fursey, "Field emission in vacuum micro-electronics" Applied Surface Science, vol. 215,pp. 113-134, (2003)

[8] L. Ozawa, and M. Itoh, "Cathode Ray Tube Phosphors" Chemical Reviews, Vol. 103,pp. 3837-3855,(2003)

[9] T. Oyama, H. Ohsaki, Y. Tachibana, et al "A new layer system of anti-reflective coating for cathode ray tubes" Thin Solid Films, vol. 351,pp. 235-240, (1999)

[10] Y. Gao, X. Zhang, W. Lei, M. Liu, et al "Ion bombardment in a normal-gate FED" Applied Surface Science, vol. 243, pp. 19-23, (2005)

[11] K. R. Shoulders, "Microelectronics using electron beam activated machining techniques" Adv. Comput., vol.2, pp.135. (1961)

[12] C. A. Spindt, "A Thin Film Field Emission Cathode" J Appl Phys, vol.39, p. 3504, (1968)

[13] C. J. Summers "Phosphors for field emission displays" Tech Digest of the 10th Int Vac Microelectron Conf, Kyongju, Korea, p. 244, (1997)

[14] G. S. Choi, K. H. Son, and D.J. Kim, "Fabrication of high performance carbon nanotube field emitters" Microelectronic Engineering, vol.66, pp. 206-212, (2003)

[15] A. Ostankov, D. Paneque, E. Lorenz, et al, "A study of the new hemispherical 6dynodes PMT from electron tubes" Nuclear Instruments and Methods in Physics Research A, vol. 442, pp. 117-123, (2000)

[16] A. J. Dekker,(1958) "Secondary Electron Emission" Solid State Physics, vol. 6, pp.251311 .

[17] O. Groning, O. M. Kuttel, Ch. Emmenegger et al "Field Emission properties of carbon nanotubes" J. Vac. Sci. Technol. B, vol. 18, pp.665-678, (2000) 
[18] M. Nikl, "Wide Band Gap Scintillation Materials: Progress in the Technology and Material Understanding" phys. stat. sol., vol. 179, pp. 595-620, (2000)

[19] J. S. Yoo and J. D. Lee, "The effects of particle size and surface recombination rate on the brightness of low-voltage phosphor" J. Appl. Phys., vol. 81, pp. 2810-2813, (1997)

[20] M. Pope, H. P. Kallmann, and P. Magnante, "Electroluminescence in Organic Crystals" J.Chem. Phys, vol. 38, pp. 2042-2043, (1963)

[21] J. H. Burroughes, D. D. C. Bradley, A. R. Brown, et al, "Light-emitting diodes based on conjugated polymers" Nature vol. 347, pp. 539-541, (1990)

[22] W. Tang, and S. A. VanSlyke, "Organic electroluminescent diodes" Appl. Phsy. Lette., vol. 51, pp. 913-915 (1987)

[23] P. Dannetun, M. Lögdlund, C. Fredriksson, et al "Reactions of low work function metals $\mathrm{Na}, \mathrm{Al}$, and $\mathrm{Ca}$ on $\alpha, \omega$ diphenyl tetra decaheptaene. Implications for metal/polymer interfaces" J. Chem. Phys., vol. 100, pp. 6765-6771.(1994)

[24] R. G. Kepler, P. M. Beeson, S. J. Jacobs et al. "Electron and hole mobility in tris(8hydroxyquinolinolato-N1,O8) aluminum" Appl. Phys. Lett., Vol. 66, pp.3618-3620, (1995)

[25] P. E. Burrows, G. Gu, V. Bulovi6 et al "Achieving full-color organic light-emitting devices for lightweight, flat-panel displays" IEEE Transactions on Electron Dev. Vol. 44, pp. 1188-1203, (1997)

[26] C. L. Lin, H. W. Lin, and C. C. Wu"Examining microcavity organic lightemittingdevices having two metal mirrors" Appl. Phys. Lett., vol. 87, pp.021101 (1-3), (2005)

[27] Y. J. Lee, S. H. K, J. Huh, et al "A high-extraction-efficiency nano patterned organic light-emitting diode" Appl. Phys. Lett., vol. 82, pp. 3779-3781,(2003)

[28] H. Kanno, Y. Sun, and S. R. Forrest,"High-efficiency top-emissive white-light-emitting organic electro-phosphorescent devices" Appl. Phys. Lett., vol. 86, pp. 263502 1-3. (2005)

[29] Y. J. Tung, M. M.-H. Lu, M. S. et al "High-Efficiency White Phosphorescent OLEDs for Lighting" Proc. of SPIE, vol. 5214, pp.114-123,(2004)

[30] Y. J. Lu, C. H. Chang, C. L. Lin, et al "Achieving three-peak white organic light-emitting devices using wavelength-selective mirror electrodes" Appl. Phys. Lett., vol. 92, pp. $123303(1-3),(2008)$

[31] C. S. Li, S. H. Su, H. Y. Chi, and M. Yokoyama "Application of highly ordered carbon nanotubes templates to field-emission organic light-emitting diodes" Journal of Crystal Growth, vol. 311, pp. 615-618, (2009)

[32] C. S. Li, S. H. Su, T. M. Lin , H. Y. Chi, and M. Yokoyama, "Luminous Efficiency Enhancement of Organic Light-Emitting Diodes by an External Electron Source", IEEE International Nano Electronics Conference (INEC).(2010)

[33] G. T. Chen, S. H. Su, and M. Yokoyama, "Field-Emission Organic Light-Emitting Device Using Oxide-Coated Cathode as Electron Source" Electrochemical and SolidState Letters, vol. 10, pp. J41-J44, (2007) 
[34] M. Pfeiffer, K. Leo, X. Zhou, et al "Doped organic semiconductors: Physics and application in light emitting diodes" Organic Electronics, vol. 4, pp. 89-103, (2003)

[35] W. A. de Heer, A. Châtelain, D. Ugarte "A Carbon Nanotube Field-Emission Electron Source" Science, Vol. 270, pp. 1179-1180, (1995)

[36] S. Iijima "Helical microtubules of graphitic carbon", Nature, vol. 354, pp. 56-58.(1991)

[37] T. 1V. Vorburger, D. Penn, and E. W. Plummer, "Field emission work functions" Surface Science, vol. 48, pp. 417-431,(1975)

[38] S. R. C. Vivekchand, L. M. Cele, F. L. Deepak, et al "Carbon nanotubes by nebulized spray pyrolysis" Chemical Physics Letters, vol. 386, pp.313-318.(2004)

[39] W. B. Choi, D. S. Chung, J. H. Kang, et al "Fully sealed, high-brightness carbonnanotube field-emission display" Appl. Phys. Lett., vol. 75, pp. 3129-3131,(1999)

[40] L. Zhu, J. Xu, Y. Xiu, et al "Growth and electrical characterization of high-aspect-ratio carbon nanotube arrays" Carbon, vol. 44, pp.253-258, (2006)

[41] Z. Wu, Z. Chen, $\mathrm{Xu} \mathrm{Du}$, et al "Transparent, Conductive Carbon Nanotube Films" Science, vol. 305, pp. 1273-1276, (2004)

[42] R. H. Schmidt, I. A. Kinloch, A. N. Burgess, and A. H. Windle "The Effect of Aggregation on the Electrical Conductivity of Spin-Coated Polymer/CarbonNanotube Composite Films" Langmuir, vol. 23, pp. 5707-5712 (2004)

[43] H. J. Jeong, H. K. Choi, G. Y. Kim, et al "Fabrication of efficient field emitters with thin multi-walled carbon nanotubes using spray method" Carbon, vol. 44, pp. 2689-2693. (2006)

[44] L.S.Hung, C.W.Tang, M.G.Mason, P.Raychaudhuri, and J. Madathil, "Application of an ultrathin $\mathrm{LiF} / \mathrm{Al}$ bilayer in organic surface-emitting diodes", J.Appl.Phys., vol.78,no.4,pp.544-546, (2000).

[45] Q.Liu, L.Duan,Y.Li,J.Qiao, Z. Yu, D. Zhang, L. Wang, G, Dong, and Y. Qiu, “Study on the electron injection mechanism of thermally decomposable Cs2CO3", Jpn. J. Appl. Phys., vol.48, pp.102302-4, October (2009).

[46] X. L. Zhu, J. X. Sun, H. J. Peng, et al "Vanadium pentoxide modified polycrystalline silicon anode for active-matrix organic light-emitting diodes" Appl Phys Lett., vol. 87, pp. 153508 (3 pages),(2005)

[47] C. H. Chen, and C. W. Tang, "Efficient green organic light-emitting diodes with stericly hindered coumarin dopants" Appl. Phys. Lett., Vol. 79, No. 22, pp.3711-3713. (2001)

[48] A. Buzulutskov, A. Breskin, and R. Chechik, "Field enhancement of the photoelectric and secondary electron emission from Csl" J. Appl. Phys. vol. 77 no.5, pp. 21382145,(1995)

[49] H. J. Jeong, H. K. Choi, G. Y. Kim, et al "Fabrication of efficient field emitters with thin multi-walled carbon nanotubes using spray method" Carbon, vol. 44, pp. 26892693,( 2006)

[50] Q. Liu, L. Duan, Y. Li, J. et al "Study on the Electron Injection Mechanism of Thermally Decomposable Cs2CO3” Jpn. J. Appl. Phys., vol. 48, pp. 102302 (4 pages), (2009) 
[51] W. A. de Heer, A. Chatelain, D. Ugarte “A Carbon Nanotube Field-Emission Electron Source" Science, vol. 270, pp. 1179-1180, (1995)

[52] Meiso Yokoyama*, Wu Chung-Ming and Shui-Hsiang Su" Enhancing the efficiency of white organic light-emitting diode using energy recyclable photovoltaic cells" Jpn. J. Appl. Phys. Vol. 51, pp.032102, (2012)

[53] J. Singh, " Developing a tandem of organic solar cell and light emitting diode" Phys. Status Solidi C 8, No. 1, 189-192 (2011) 